\title{
A SPECIAL RELATIVISTIC HEAT ENGINE
}

\section{WILLIAM S. CARIENS}

\author{
12550 Lake Avenue \\ Lakewood, Ohio 44107 \\ (Received April 28, 1982)
}

\begin{abstract}
This paper offers a marriage of concepts from both classsical thermodynamics and special relativity, thus forming a new part of modern thermodynamics. The main concepts taken from themodynamics and special relativity are those of a heat engine and $E=\mathrm{mc}^{2}$, respectively. Central to understanding the operation of this relativistic heat engine is the fact that upon heating a mass, its rest mass increases! This concept is nonexistent in classical thermodynamics. An increase in rest mass means that both the internal energy of a mass and its macroscopic kinetic energy increase!!!
\end{abstract}

Thus, it is seen that special relativity provides a way to upgrade immediately part of a low form of energy, heat, into a high quality energy, macroscopic kinetic energy. This was the genesis of this engine.

Here is the engine's operation: Accelerate a mass to a high speed. At that speed add heat from a comoving heat reservoir, thus increasing the kinetic energy of the mass. After heating, slow the "heavier" mass down to its initial speed. More work is seen to be produced during the last process than was absorbed during acceleration. Next, cool the mass to complete the cycle.

The efficiency of this engine is derived, and seen to be analogous to the corresponding Carnot equation.

\section{INTRODUCTION.}

Let $u$ be the speed of a mass in the observer's (the reader's) inertial frame of reference, and let $\mathrm{c}$ be the speed of light in vacuum. Furthermore, let the subscripts $\mathrm{L}$ and $\mathrm{H}$ mean lower and higher, respectively. 
Imagine an object that is accelerated from $(u / c)_{L}$ to $(u / c)_{H}$. Next, heat the object, after which it is more massive, Pauli (1). Now, decelerate the object back to $(u / c)_{L}$. Note that the positive work involved during deceleration is greater than the absolute value of the negative work involved during acceleration. This is due to the fact that the mass is greater at the beginning of the slowing down process than it was at the end of the speeding up process. (A hot brick has more mass than the same brick at a lower temperature, Pauli (1)).

If these processes are augmented with a cooling process, one has a heat engine cycle. This cycle will be examined with more rigor in section 2.

The efficiency of the engine discussed herein is analogous to the equation for Carnot efficiency.

2. THE ENGINE.

One of the classical definitions of a heat engine is: A HEAT ENGINE is a (1) closed, (2) stationary thermodynamic system which (3) receives heat and produces work while executing a (4) cycle. The modern definition of a heat engine which is proposed here is the same as the definition above, but with condition (2) deleted.

The Minkowski energy-momentum diagram that depicts the special relativistic heat engine cycle is given in Figure 1.

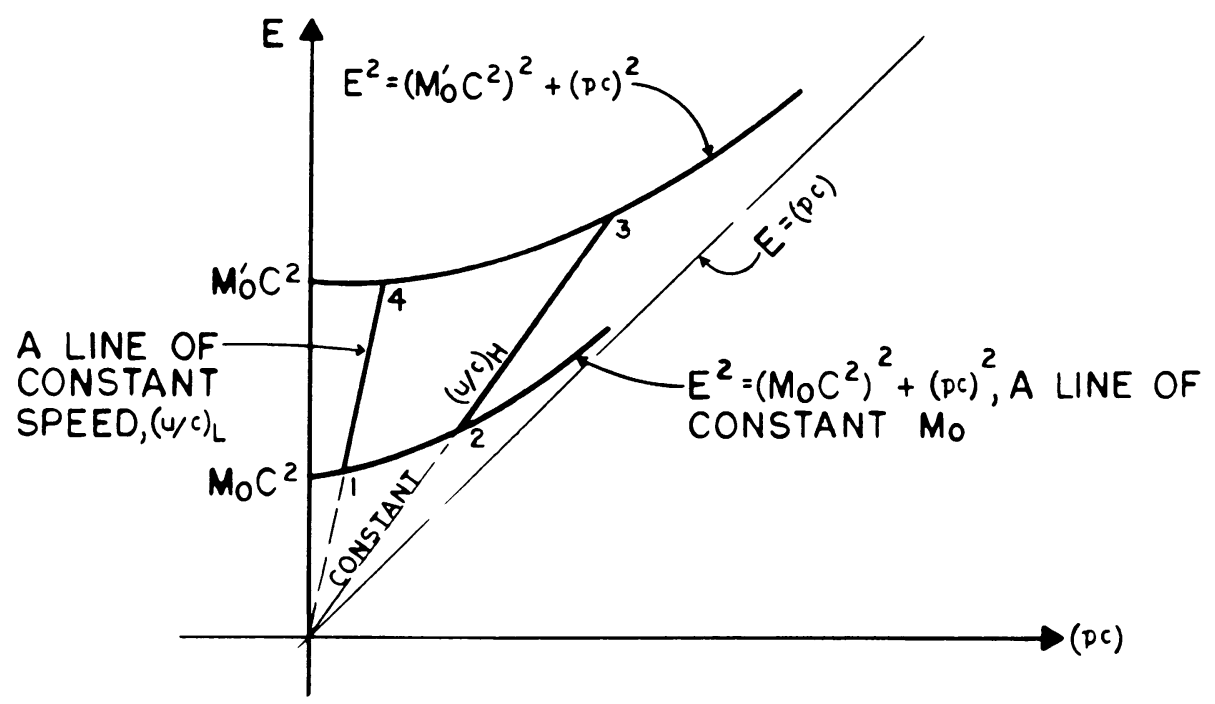

Figure 1. Heat Engine Cycle 1-2-3-4-1. 
In Figure 1, $\mathrm{E}$ is the total energy of the system, $\mathrm{p}$ is the momentum of the system, $m_{0}$ is the system's rest mass at states 1 and 2 , and $m_{0}^{\prime}$ is the system's rest mass at states 3 and 4 .

Define $\gamma$, the Lorentz factor, by

$$
\gamma \equiv\left[1-(\mathrm{u} / \mathrm{c})^{2}\right]^{-\frac{1}{2}}
$$

Then, from special relativity, the energy is given by

$$
E=r m_{0} c^{2}
$$

See Bergmann (2). Equation (2.2) will be used to analyze the engine's four processes as well as to derive the thermal efficiency of the cycle.

This engine is a closed thermodynamic system that receives heat and produces work while executing the cycle depicted in Figure 1.

3. THE PROCESSES IN FIGURE 1 .

In process 1-2, the system is accelerated adiabatically at constant rest mass, $\mathrm{m}_{\mathrm{o}}$. Let $\mathrm{W}$ and $\mathrm{Q}$ be the thermodynamic work and heat, respectively. Then equation (2.2) and the First Law of Thermodynamics, $Q-W=\Delta E$, yield

$$
-W_{1-2}=\Delta E=(\Delta \gamma) m_{0} c^{2}
$$

therefore

$$
w_{1-2}=\left(\gamma_{L}-\gamma_{H}\right) m_{0} c^{2}<0
$$

In process 2-3, the system is heated at constant speed (constant $\gamma$ ) from state 2 to state 3. Then equation (2.2) and the First Law give

$$
Q_{2-3}=\Delta E=\gamma_{H}\left(\Delta m_{0}\right) c^{2}
$$

therefore

$$
Q_{2-3}=\gamma_{H}\left(m_{0}^{\prime}-m_{0}\right) c^{2}>0
$$

See Lawden (3).

In process $3-4$, the system is decelerated adiabatically at constant rest mass, $\mathrm{m}_{\mathrm{o}}^{\prime}$. Equation $(2,2)$ and the First Law yield

$$
-\mathrm{W}_{3-4}=\Delta \mathrm{E}=-(\Delta \gamma) \mathrm{m}_{0}^{\prime} \mathrm{c}^{2}
$$

therefore

$$
w_{3-4}=\left(\gamma_{H}-\gamma_{L}\right) m_{O}^{\prime} c^{2}>0 \text {. }
$$

In process 4-1, the system is cooled at constant speed (constant $\gamma$ ) from state 4 back to initial state 1. This completes the cycle. Equation (2.2) and the First Law give 


$$
\begin{array}{ll}
Q_{4-1}=\Delta E=-\gamma_{L}\left(\Delta m_{0}\right) c^{2} \\
\text { therefore } & Q_{4-1}=\gamma_{L}\left(m_{0}-m_{0}^{\prime}\right) c^{2}<0 .
\end{array}
$$

4. THE EFFICIENCY.

The definition of thermal efficiency, $n$, yields

Hence,

$$
\eta \equiv \frac{W}{Q_{2-3}}=\frac{Q_{2-3}+Q_{4-1}}{Q_{2-3}}
$$

Substitute equations (3.2) and (3.4) into equation (4.2) to get

$$
n=1+\frac{\gamma_{L}\left(m_{O}-m_{o}^{\prime}\right) c^{2}}{\gamma_{H}\left(m_{O}^{\prime}-m_{o}\right) c^{2}}
$$

Therefore,

$$
\eta=1+\frac{Q_{4-1}}{Q_{2-3}}
$$

$$
\eta=1-\frac{\gamma_{1}}{\gamma_{H}}
$$

iotice that equation (4.4) is analogous to the corresponding Carnot equation which is given by

$$
\eta_{c}=1-\frac{T_{L}}{T_{H}}
$$

See Van Wylen (4) and Zemansky (5). Furthermore, the Lorentz factor is seen to be analogous to absolute temperature.

\section{REFERENCES}

1. PAULI, W. Theory of Relativity, Peramon Press, New York, (1958), 121-123.

2. BERGMAiN, P. G. Introduction to the Theory of Relativity, Dover Publications, Inc., New York, (1976), 92-93.

3. LAWDEN, D. F. Tensor Calculus and Relativity, Chapman and Hall, London, (1975), 48-49.

4. VAN WYLEN, G. J., SONNTAG, R. E. Fundamentals of Classical Thermodynamics, John Wiley and Sons, Inc., New York, (1978), 166-186 and Chapter 9, Second Edition S. I. Version.

5. ZEMANSKY, M. W. Heat and Thermodynamics, McGraw-Hill, New York, (1957), Fourth Edition, 139-140 and Chapter 9. 


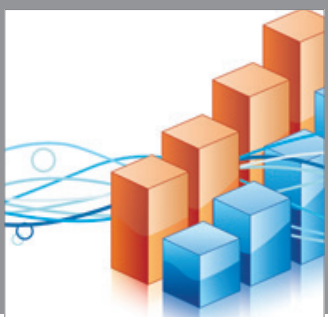

Advances in

Operations Research

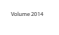

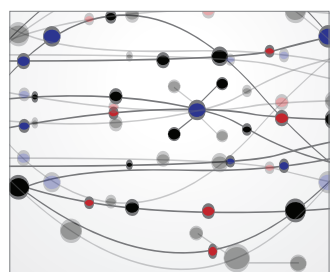

\section{The Scientific} World Journal
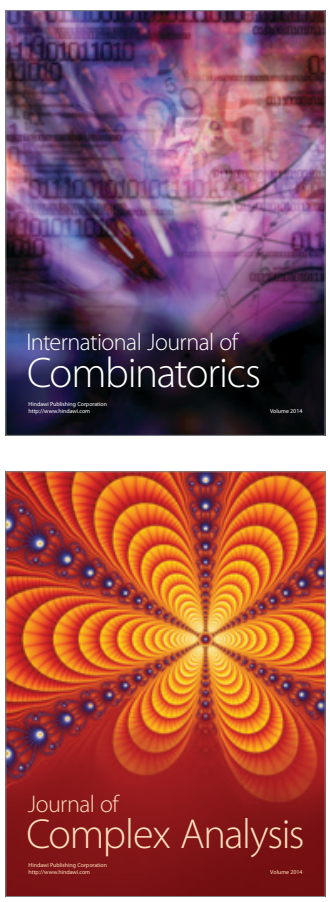

International Journal of

Mathematics and

Mathematical

Sciences
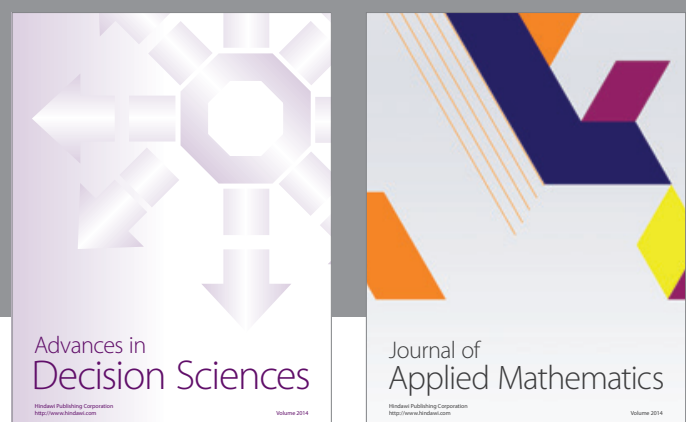

Journal of

Applied Mathematics
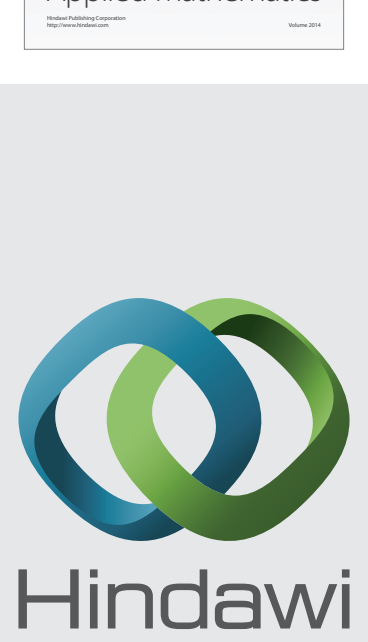

Submit your manuscripts at http://www.hindawi.com
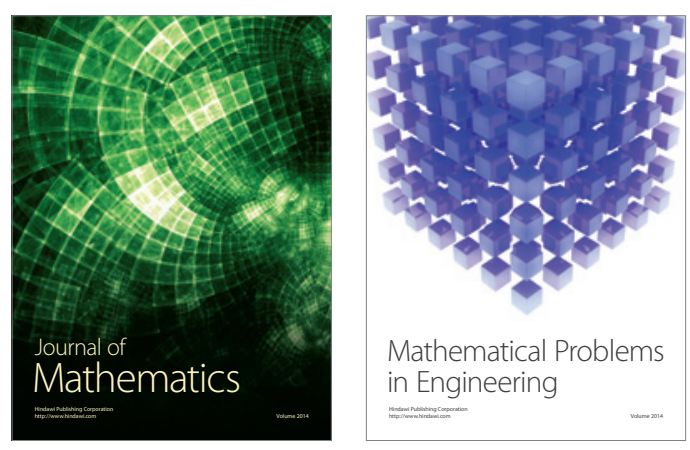

Mathematical Problems in Engineering
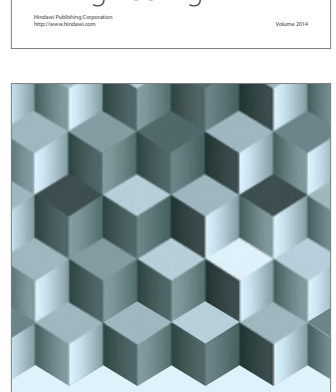

Journal of

Function Spaces
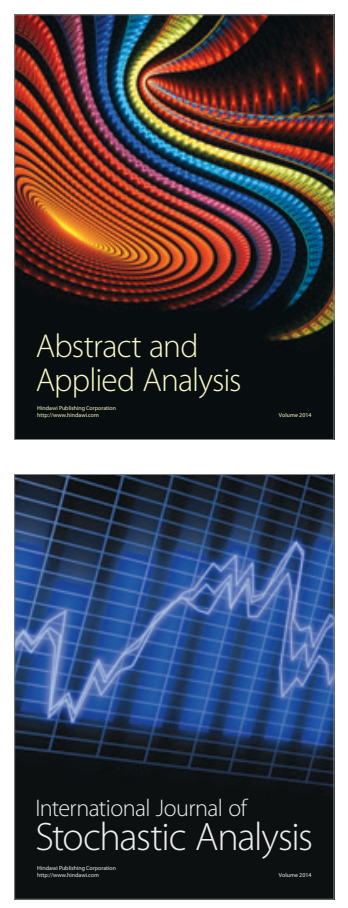

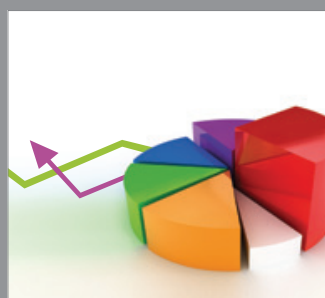

ournal of

Probability and Statistics

Promensencen
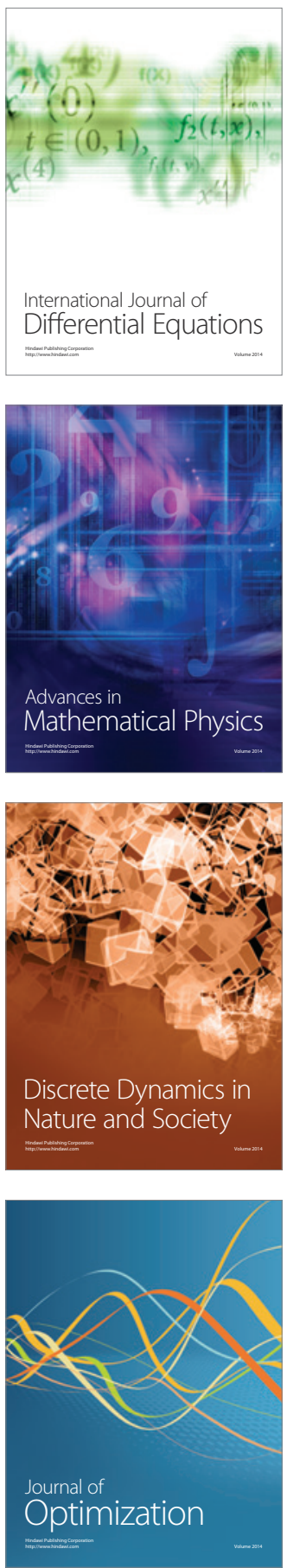